\title{
Revisiting a theorem by Folkman on graph colouring
}

\author{
Marthe Bonamy* \\ Service public français de la recherche \\ CNRS, LaBRI \\ Université de Bordeaux, France \\ marthe. bonamy@u-bordeaux.fr \\ Oscar Defrain
}

Service public français de la recherche

LIMOS, CNRS

Université Clermont Auvergne

Clermont-Ferrand, France

oscar.defrain@uca.fr

\author{
Pierre Charbit ${ }^{\dagger}$
}

Service public français de la recherche Université Paris Diderot - IRIF

Paris, France

charbit@irif.fr

Gwenaël Joret ${ }^{\S}$

Département d'Informatique Université Libre de Bruxelles

Bruxelles, Belgique

gjoret@ulb.ac.be

\author{
Aurélie Lagoutte Vincent Limouzy $^{\ddagger} \quad$ Lucas Pastor ${ }^{\ddagger}$ \\ Service public français de la recherche \\ LIMOS, CNRS \\ Université Clermont Auvergne \\ Clermont-Ferrand, France \\ \{aurelie.lagoutte, vincent.limouzy, lucas.pastor\}@uca.fr \\ Jean-Sébastien Sereni \\ Service public français de la recherche \\ Centre national de la recherche scientifique \\ ICube (CSTB), Strasbourg, France. \\ sereni@kam.mff.cuni.cz
}

Submitted: Jul 26, 2019; Accepted: Feb 1, 2020; Published: Mar 20, 2020

(C) The authors. Released under the CC BY-ND license (International 4.0).

\footnotetext{
* Supported by ANR Project DISTANCIA (ANR-17-CE40-0015) operated by the French National Research Agency (ANR).

†Supported by ANR Project DISTANCIA (ANR-17-CE40-0015), ANR Project HOSIGRA (ANR-17CE40-0022) operated by the French National Research Agency (ANR) and by INRIA GANG Project team.

${ }^{\ddagger}$ Supported by ANR Project GRAPHEN (ANR-15-CE40-0009) operated by the French National Research Agency (ANR).

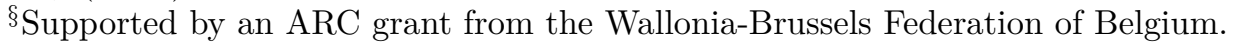




\begin{abstract}
We give a short proof of the following theorem due to Jon H. Folkman (1969): The chromatic number of any graph is at most 2 plus the maximum over all subgraphs of the difference between the number of vertices and twice the independence number.
\end{abstract}

Mathematics Subject Classifications: 05C15

\title{
1 Introduction
}

Independent sets in a graph count among the most studied objects of graph theory, both for their theoretical and practical appeal. An independent set in a graph is a set of vertices no two of which are adjacent. This notion is at the heart of graph colouring, where one tries to find a partition of the vertex set of a graph in the smallest possible number of independent sets, this number being the chromatic number of the graph. It follows that every graph $G$ with chromatic number $k$ has an independent set of size at least $|V(G)| / k$. We investigate what kind of converse statement could be true. As is usual, we write $\chi(G)$ for the chromatic number of $G$ and $\alpha(G)$ for the independence number of $G$, that is, the size of a largest independent set in $G$. Since we study the independence number of a graph in relation to the number of its vertices, it is useful to define the independence ratio $\operatorname{ir}(G)$ of a graph $G$ (with at least one vertex) to be $\frac{\alpha(G)}{|V(G)|}$, and the minimum independence ratio $\operatorname{mir}(G)$ to be $\min \{\operatorname{ir}(H): H$ induced subgraph of $G$ with at least one vertex $\}$. (The inverse of the minimum independence ratio is sometimes called the Hall ratio.) Let us start with a straightforward observation. Every graph with chromatic number greater than 2 contains an induced odd cycle, and the independence number of an odd cycle is less than half the number of its vertices. The contrapositive statement reads as follows.

Observation 1. If every induced subgraph $H$ of a graph $G$ has independence ratio at least $\frac{1}{2}$, then $G$ has chromatic number at most 2 .

One could try to generalise Observation 1 in several ways. For instance, what about replacing the constant 2 by some larger integer $k$ ? This would yield an incorrect statement, as for each integer $k \geqslant 3$, there is a graph with chromatic number greater than $k$ and minimum independence ratio at least $\frac{1}{k}$. Indeed, let $M_{k}$ be the $k^{\text {th }}$ Mycielski graph (so $M_{2}$ is a cycle of length 5). Then $\chi\left(M_{k}\right)=k+1$ and, as is well known, every proper subgraph of $M_{k}$ has chromatic number at most $k$, from which one infers that $\operatorname{mir}\left(M_{k}\right) \geqslant \frac{1}{k}$ whenever $k \geqslant 3$.

Let us point out here that the exact value of the minimum independence ratio of Mycielski graphs seems to be unknown. In 2006, Cropper, Gyárfás and Lehel [CGL06] proved that every triangle-free graph is an induced subgraph of a Mycielski graph and, using results on Ramsey numbers [AKS80, Kim95] and on the fractional chromatic number of Mycieslki graphs [LPU95], they inferred the existence of two positive real numbers $c_{1}$ and $c_{2}$ such that for every integer $m \geqslant 3$,

$$
c_{1} \cdot \frac{\sqrt{\log m}}{m} \leqslant \operatorname{mir}\left(M_{s}\right) \leqslant c_{2} \cdot \frac{\log m}{m} .
$$


Here $s$ is one less than the Ramsey number $R(3, m)$, which is the largest integer $n$ such that there exists an $n$-vertex triangle-free graph with independence number less than $m$. As is well known, $R(3, m)=\Theta\left(m^{2} / \log m\right)$ (the lower bound was established by Kim [Kim95] in 1995, while the upper bound had been proved fifteen years before by Ajtai, Komlós and Szemerédi [AKS80]; simpler proofs for the lower bound along with improvements of the multiplicative constant were found subsequently [AKS81, Gri83, She91]).

Another way is to look for approximate generalisations of Observation 1. Let us say that a graph is half-stable if its independence ratio is at least one half.

Question 2. Let $k$ be a non-negative integer and $G$ a graph. Assume that for every induced subgraph $H$ of $G$, there exists a set $Y \subseteq V(H)$ of at most $k$ vertices such that $H-Y$ is half-stable. Is it true that there exists a subset $X \subseteq V(G)$ of at most $k$ vertices such that $G-X$ is bipartite?

Again this turns out to be false: for $k=1$, consider the graph obtained from the cycle $v_{1} v_{2} v_{3} v_{4} v_{5} v_{6}$ of length 6 by adding the triangle $v_{1} v_{3} v_{5}$ (see Figure 1 ).

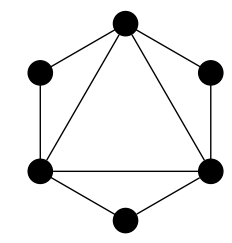

Figure 1: A negative answer to Question 2 for $k=1$.

A more careful question was raised by Erdős and Hajnal. A graph satisfying the hypothesis of Question 2 has been called k-near-bipartite in the literature [Ree99]. Erdös and Hajnal conjectured (see [Gyá97]) the existence of a function $g: \mathbf{N} \rightarrow \mathbf{N}$ such that every $k$-near-bipartite graph $G$ contains a set $X$ of at most $g(k)$ vertices such that $G-X$ is bipartite. This conjecture was confirmed by Reed [Ree99] in 1999.

Here is yet another way to weaken Question 2: it was raised by Erdős and Hajnal [EH68] and gave rise to a "deep theorem" (to quote Gyárfás [Gyá97]) demonstrated by Folkman [Fol70] in 1969.

Theorem 3 (Folkman [Fol70]). Let $k$ be a non-negative integer and $G$ a graph. If for every induced subgraph $H$ of $G$, there exists a set $Y \subseteq V(H)$ of at most $k$ vertices such that $H-Y$ is half-stable, then $\chi(G) \leqslant k+2$.

The arguments developed by Folkman to demonstrate Theorem 3 are nice and interesting. However, they are difficult to access and it seems they deserve more visibility. The essence of Folkman's argument is not obvious to see amongst the seventeen lemmas composing its proof, which is witnessed by the fact that a simpler proof was asked for by Claude Tardif at a workshop at the University of Illinois in 2008 [REG08]. Our goal is to present a much more compact and shorter proof of Theorem 3, which still relies on Folkman's original idea, but with refined, factorised and sometimes generalised statements. 
We define the potential $\rho(H)$ of a graph $H$ to be $|V(H)|-2 \alpha(H)+2$. Note that if a graph has potential $k+2$ then one can remove $k$ vertices (but not fewer) to obtain a half-stable graph. Given a graph $G$, let $f(G)$ be the maximum of $\rho(H)$ over all induced subgraphs $H$ of $G$. Note that if an induced subgraph $H$ satisfies the property mentioned in Theorem 3, then $\rho(H) \leqslant k+2$, and hence Theorem 3 can be reformulated as $\chi(G) \leqslant f(G)$ for every graph $G$. An induced subgraph $H$ of $G$ is a witness if $\rho(H)=f(G)$. A $k$-colouring of a graph $G$ is a mapping $\varphi: V(G) \rightarrow\{1,2, \ldots, k\}$ such that no two adjacent vertices have the same image under $\varphi$. If $X$ is a subset of vertices of a graph $G$, then $G[X]$ is the subgraph of $G$ induced by the vertices in $X$ and $G-X$ is the subgraph of $G$ induced by the vertices not in $X$.

To demonstrate Theorem 3, we use a simple, seemingly unrelated result, which is a special case of a theorem proved by Hajnal [Haj65] in 1965. A proof is included for completeness (the formulation and the argument are different from what is customary seen).

Proposition 4 (Hajnal [Haj65]). If a graph $G$ admits an independent set containing more than half of the vertices, then there exists a vertex contained in all maximum independent sets of $G$.

Proof. We proceed by induction on the number $e$ of edges of $G$. The statement is true if $e$ is zero. Assume that $e \geqslant 1$ and the statement is true for graphs with fewer than $e$ edges. Note that the statement is true if $G$ has an isolated vertex, and hence we may assume by induction that $G$ is connected. If there is an edge $e$ such that $\alpha(G-e)=\alpha(G)$, then by induction a vertex $v$ is contained in all maximum independent sets of $G-e$, and hence also in all those of $G$ since each of them is also a maximum independent set of $G-e$. Thus it is enough to show that such an edge $e$ exists. Arguing by contradiction, let us assume that $\alpha(G-e)>\alpha(G)$ for every edge $e$ of $G$.

Let $x$ be a vertex of $G$ contained in the largest number of maximum independent sets of $G$. Let $G^{\prime}$ be the graph obtained from $G$ by deleting $x$ and all its neighbours. We show that the induction hypothesis can be applied to $G^{\prime}$. Notice that adding $x$ to any independent set of $G^{\prime}$ yields an independent set of $G$, and hence $\alpha\left(G^{\prime}\right) \leqslant \alpha(G)-1$. It follows that $\alpha\left(G^{\prime}\right)=\alpha(G)-1$ because $x$ is contained in at least one maximum independent set of $G$. Since $G$ is connected and has more than one vertex, we know that $\left|V\left(G^{\prime}\right)\right| \leqslant|V(G)|-2$, and therefore the induction hypothesis applies to $G^{\prime}$, ensuring the existence of a vertex $y$ of $G^{\prime}$ contained in every maximum independent set of $G^{\prime}$. Consequently, $y$ is contained in every maximum independent set of $G$ that contains $x$. The definition of $x$ thus ensures that a maximum independent of $G$ contains $x$ if and only if it contains $y$.

To conclude, let $z$ be a neighbour of $x$. Since $\alpha(G-x z)>\alpha(G)$, there exists a maximum independent set $I_{x}$ of $G$ that contains $x$ and such that the only neighbour of $z$ in $I_{x}$ is $x$. However, this implies that $\left(I_{x} \backslash\{x\}\right) \cup\{z\}$ is a maximum independent set of $G$ containing $y$ and not $x$, a contradiction. This concludes the proof. 


\section{Proof of Theorem 3}

We proceed by contradiction. Let $G$ be a counter-example to Theorem 3 with the fewest vertices. In particular, $\chi(G) \geqslant f(G)+1$. If $f(G)=2$, Observation 1 implies that $G$ is bipartite, a contradiction. It follows that $f(G) \geqslant 3$, and hence $\chi(G) \geqslant 4$.

Note that every proper induced subgraph of $G$ admits a $(\chi(G)-1)$-colouring. We argue below that the graph satisfies a deeper critical property.

(A). For every subset $X$ of vertices of $G$,

$$
\chi(G) \geqslant \chi(G[X])+\chi(G-X)-1 .
$$

In particular, (A) implies that removing a clique of size $p$ in $G$ results in a graph with chromatic number either $\chi(G)-p$ or $\chi(G)-p+1$.

Proof of (A). The statement holds trivially if $X$ or $G-X$ is empty. Therefore, both $G_{1}:=$ $G[X]$ and $G_{2}:=G-X$ have fewer vertices than $G$. The minimality of $G$ thus implies that none of $G_{1}$ and $G_{2}$ is a counter-example to the statement of Theorem 3. For each $i \in\{1,2\}$, let $H_{i}$ be a witness of $G_{i}$. We have $\rho\left(H_{i}\right)=f\left(G_{i}\right) \geqslant \chi\left(G_{i}\right)$. We observe that $f(G) \geqslant \rho\left(G\left[V\left(H_{1}\right) \cup V\left(H_{2}\right)\right]\right) \geqslant \rho\left(H_{1}\right)+\rho\left(H_{2}\right)-2$, and hence $f(G) \geqslant \chi\left(G_{1}\right)+\chi\left(G_{2}\right)-2$. Since $\chi(G) \geqslant f(G)+1$ by assumption, the conclusion follows.

(B). The graph $G$ has no induced even cycle.

Proof. We proceed by contradiction. Assume that $C$ is an induced even cycle of $G$, and let $2 p$ be its length. Let $A$ and $B$ be the two maximum independent sets of $C$. We consider the graph $G^{\prime}$ obtained from $G$ by merging all vertices of $A$ in a single vertex $a$ and all vertices of $B$ in a single vertex $b$, replacing every multi-edge that arises by a single edge (see Figure 2).

Note that $G^{\prime}$ has fewer vertices than $G$ and thus satisfies the conclusion of Theorem 3 . It follows that

$$
f\left(G^{\prime}\right) \geqslant \chi\left(G^{\prime}\right) \geqslant \chi(G) \geqslant f(G)+1 .
$$

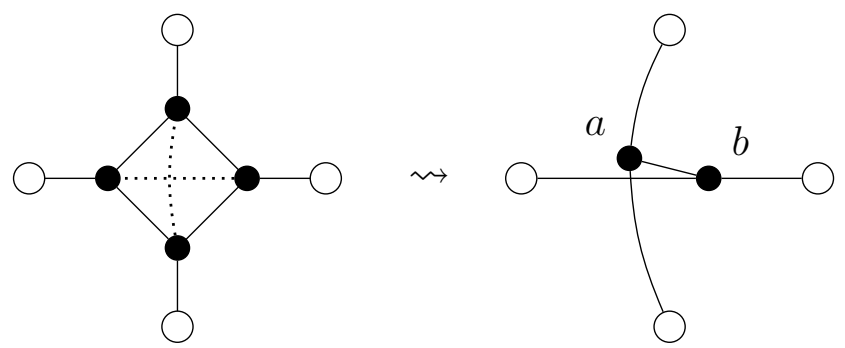

Figure 2: An example of the reduction from $G$ to $G^{\prime}$ for (B). 
Let $H^{\prime}$ be a witness of $G^{\prime}$, so $\rho\left(H^{\prime}\right) \geqslant f(G)+1$. Let us consider the subgraph $H$ of $G$ induced by $\left(V\left(H^{\prime}\right) \backslash\{a, b\}\right) \cup V(C)$. The potential $\rho(H)$ of $H$ can be bounded as follows.

$$
\begin{aligned}
f(G) & \geqslant \rho(H) \\
& \geqslant \rho\left(H^{\prime}\right)+|V(C)|-2-2\left(\alpha(H)-\alpha\left(H^{\prime}\right)\right) \\
& \geqslant f(G)+2 p-1-2\left(\alpha(H)-\alpha\left(H^{\prime}\right)\right) .
\end{aligned}
$$

Clearly, $\alpha(H)-\alpha\left(H^{\prime}\right) \leqslant \alpha(C)=p$. In fact, we must have $\alpha(H)-\alpha\left(H^{\prime}\right) \leqslant p-1$, as we now explain. Let $I$ be a maximum independent set of $H$. If $|I \cap V(C)| \leqslant p-1$, then $I \cap V\left(H^{\prime}\right)$ is an independent set of $H^{\prime}$ of size at least $\alpha(H)-(p-1)$. Otherwise, $I \cap V(C) \in\{A, B\}$, and hence one of $(I \backslash V(C)) \cup\{a\}$ and $(I \backslash V(C)) \cup\{b\}$ is an independent set of $H$ of size at least $\alpha(H)-(p-1)$.

Plugging the inequality $\alpha(H)-\alpha\left(H^{\prime}\right) \leqslant p-1$ into (1) yields that

$$
f(G) \geqslant f(G)+2 p-1-2(p-1)>f(G),
$$

which is the desired contradiction.

We call diamond the graph obtained from the complete graph on 4 vertices by deleting an edge (see the graph induced by $u, v, x, y$ in Figure 3 ).

(C). No induced subgraph of $G$ is a diamond.

Proof. Let $x y$ be an edge of $G$. Let $A$ be the set of common neighbors of $x$ and $y$, and assume for a contradiction that $A$ does not induce a clique. For every two distinct vertices $u$ and $v$ in $A$ such that $u v \notin E(G)$, let $G_{u v}$ be the graph obtained from $G-\{x, y\}$ by merging the two vertices $u$ and $v$ into a vertex, once again replacing every multi-edge that arises by a single edge (see Figure 3 ).

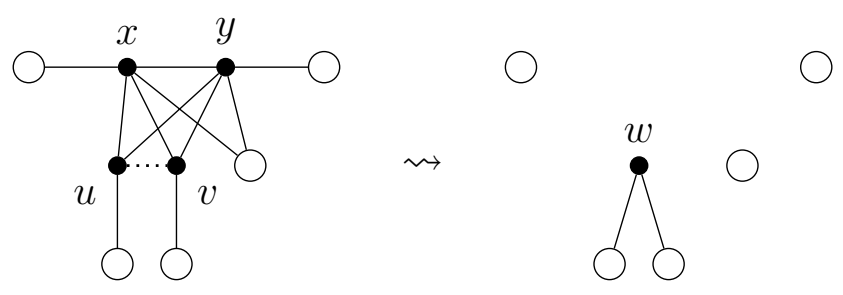

Figure 3: An example of the reduction from $G$ to $G_{u v}$ for (C).

We prove the following statement.

(C1). For every two distinct vertices $u$ and $v$ in $A$ that are not adjacent in $G$,

(1) $\chi\left(G_{u v}\right)=\chi(G)-2$; and

(2) in every $(\chi(G)-2)$-colouring of $G_{u v}$, all colours appear on $A$. 
Proof. Let $w$ be the vertex resulting from the identification of $u$ and $v$. We first prove (1). Let $H$ be a witness of $G_{u v}$. If $H$ contains $w$, then we let $H^{\prime}$ be the subgraph of $G$ induced by $(V(H) \backslash\{w\}) \cup\{u, v, x, y\}$. If $H$ does not contain $w$, then we let $H^{\prime}$ be the subgraph of $G$ induced by $V(H) \cup\{u, x, y\}$. In either case, we observe that $\rho\left(H^{\prime}\right)>\rho(H)$ because $\alpha\left(H^{\prime}\right) \leqslant \alpha(H)+1$. Consequently, we derive that $f\left(G_{u v}\right) \leqslant f(G)-1 \leqslant \chi(G)-2$.

Because $G_{u v}$ has fewer vertices than $G$, we have $f\left(G_{u v}\right) \geqslant \chi\left(G_{u v}\right)$, and hence $\chi\left(G_{u v}\right) \leqslant$ $\chi(G)-2$. On the other hand, $\chi\left(G_{u v}\right) \geqslant \chi(G)-2$ also holds (from a $\chi\left(G_{u v}\right.$ )-colouring of $G_{u v}$, it suffices to keep the same colour on the vertices belonging to both graphs, assign the colour of $w$ to each of $u$ and $v$, and the colours $\chi\left(G_{u v}\right)+1$ and $\chi\left(G_{u v}\right)+2$ to $x$ and $y$, respectively, to obtain a $\left(\chi\left(G_{u v}\right)+2\right)$-colouring of $\left.G\right)$. Therefore, $\chi\left(G_{u v}\right)=\chi(G)-2$.

Let us now prove (2). Suppose, on the contrary, that there is a $(\chi(G)-2)$-colouring $\varphi$ of $G_{u v}$ contradicting (2), and let $c \in \varphi\left(V\left(G_{u v}\right)\right) \backslash \varphi(A)$. We derive from $\varphi$ a $(\chi(G)-1)$ colouring of $G$, which is a contradiction. Set $c^{\prime}=\chi(G)-1$, so $c^{\prime} \notin \varphi\left(V\left(G_{u v}\right)\right)$. We define $\phi$ to be the colouring of $G$ obtained from $\varphi$ by colouring $u$ and $v$ with $\varphi(w)$, colouring $x$ with $c$ and $y$ with $c^{\prime}$, and changing the colour of every neighbour of $x$ that belongs to $\varphi^{-1}(\{c\})$ to $c^{\prime}$. As $\varphi^{-1}(\{c\})$ is an independent set disjoint from $A$, and all common neighbours of $x$ and $y$ belong to $A$, we infer that $\phi$ is a proper $(\chi(G)-1)$-colouring of $G$.

We now consider the graph $G^{\prime}$ obtained from $G-\{x, y\}$ by creating a vertex $z$ with neighbourhood $A$. We observe that the existence of a $(\chi(G)-2)$-colouring of $G^{\prime}$ would contradict (C1). Indeed, (C1) implies that $|A| \geqslant \chi(G)-1$. It follows that if $\varphi$ is a $(\chi(G)-2)$-colouring of $G^{\prime}$, then there exist two vertices $u$ and $v$ in $A$ such that $\varphi(u)=\varphi(v)$. Consequently, $u$ and $v$ are not adjacent in $G$ and thus $\varphi$ readily yields a $(\chi(G)-2)$-colouring of $G_{u v}$ such that one colour, namely $\varphi(z)$, does not appear on $A$. Therefore, $\chi\left(G^{\prime}\right) \geqslant \chi(G)-1$, and hence $f\left(G^{\prime}\right) \geqslant \chi\left(G^{\prime}\right) \geqslant f(G)$.
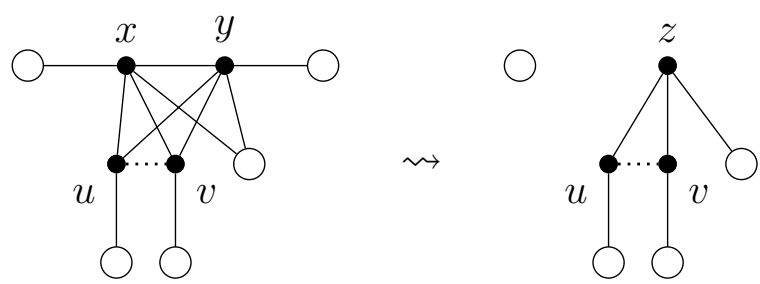

Figure 4: An example of the reduction from $G$ to $G^{\prime}$ for $(\mathrm{C})$.

Let $H^{\prime}$ be a witness of $G^{\prime}$, so $\rho\left(H^{\prime}\right) \geqslant f(G)$. If $z \in V\left(H^{\prime}\right)$, then the potential of the subgraph $H_{0}$ of $G$ induced by $\left(V\left(H^{\prime}\right) \backslash\{z\}\right) \cup\{x, y\}$ is larger than that of $H^{\prime}$ because $\alpha\left(H_{0}\right) \leqslant \alpha\left(H^{\prime}\right)$. This is a contradiction since $f(G) \leqslant f\left(G^{\prime}\right)=\rho\left(H^{\prime}\right)$. In particular, $H^{\prime}$ is thus an induced subgraph of $G-\{x, y\}$ and consequently we deduce that $f(G)=\rho\left(H^{\prime}\right)$. We also deduce that the potential of the subgraph of $G^{\prime}$ induced by $V\left(H^{\prime}\right) \cup\{z\}$ is less than that of $H^{\prime}$, so there exists a maximum independent set of $H^{\prime}$ that is disjoint from $A$. If there is a vertex $w \in A \backslash V\left(H^{\prime}\right)$, then the potential of the subgraph of $G$ induced by $V\left(H^{\prime}\right) \cup\{w, x, y\}$ is larger than that of $H^{\prime}$, a contradiction. Therefore $A \subseteq V\left(H^{\prime}\right)$, and $z \notin V\left(H^{\prime}\right)$. 
Let $U=V\left(H^{\prime}\right) \backslash A$. We note that $|U|=\left|V\left(H^{\prime}\right)\right|-|A|$. From (C1), we derive that $|A| \geqslant \chi(G)-1 \geqslant f(G)=\rho\left(H^{\prime}\right)$. Therefore, $|U| \leqslant 2 \alpha\left(H^{\prime}\right)-2$. Set $G_{U}=H^{\prime}-A$, so $G_{U}$ is the subgraph of $G$ induced by $U$. It follows from the previous paragraph that $\alpha\left(G_{U}\right)=\alpha\left(H^{\prime}\right)$, which is greater than $\frac{1}{2} \cdot|U|$. Proposition 4 therefore implies the existence of a vertex $w$ in $U$ such that every independent set of $G_{U}$ that has size $\alpha\left(H^{\prime}\right)$ contains $w$.

We consider the subgraph $H$ of $G$ induced by $\left(V\left(H^{\prime}\right) \backslash\{w\}\right) \cup\{x, y\}$. Since its potential is at most $f(G)$, there is a maximum independent set $I$ in $H$ of size $\alpha\left(H^{\prime}\right)+1$. We observe that $I$ contains exactly one of $\{x, y\}$, say $x$ without loss of generality. We derive that $I \backslash\{x\}$ is disjoint from $A$, which is contained in the neighbourhood of $x$. We note that $I \backslash\{x\}$ is an independent set of size $\alpha\left(H^{\prime}\right)=\alpha\left(G_{U}\right)$ that is contained in $U$ and does not contain $w$, a contradiction.

Equipped with all these properties, we may now finish the proof of Theorem 3 . We start by proving that $G$ has no triangle. Choose a maximum clique $K$ of $G$, and let $\omega$ be its size. We let $u_{1}, u_{2}, \ldots, u_{\omega}$ be the vertices of $K$ and we set $G_{K}=G-K$. For every $i \in\{1,2, \ldots, \omega\}$, we let $N_{i}$ be the neighbourhood of $u_{i}$ in $G_{K}$. By (C), and because $K$ is maximum, the sets $\left(N_{i}\right)_{1 \leqslant i \leqslant \omega}$ are pairwise disjoint. Moreover, (B) implies that they are pairwise non-adjacent, that is, if $G$ has an edge with one end-vertex in $N_{i}$ and the other in $N_{j}$, then $i=j$.

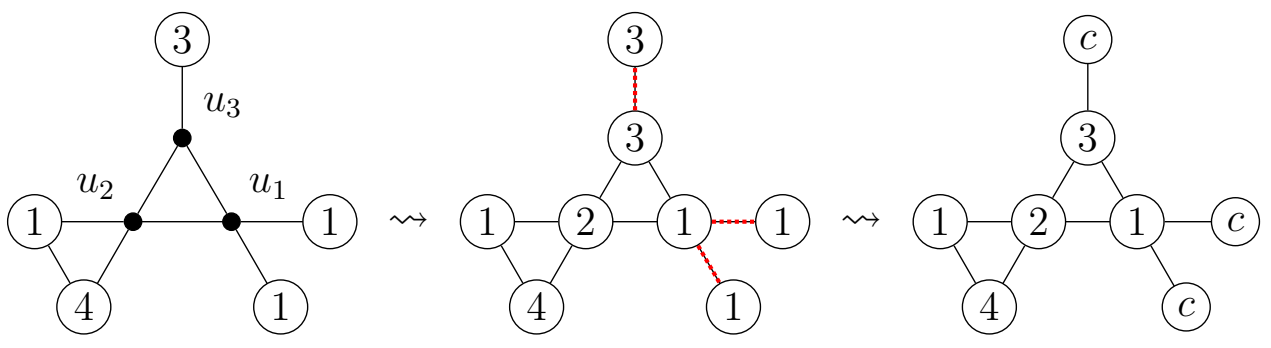

Figure 5: An example of the colouring extension when $\omega(G) \geqslant 3$.

Suppose, for a contradiction, that $\omega \geqslant 3$. We deduce from $(\mathrm{A})$ that $\chi\left(G_{K}\right) \leqslant \chi(G)-2$. Let $\varphi$ be a $(\chi(G)-2)$-colouring of $G_{K}$. We argue how to obtain from $\varphi$ a $(\chi(G)-1)$ colouring of $G$, which would be a contradiction. Set $c=\chi(G)-1$, so $c \notin \varphi\left(V\left(G_{K}\right)\right)$. Colour every vertex $v$ not in $K$ with $\varphi(v)$. For each $i \in\{1,2, \ldots, \omega\}$, colour $u_{i}$ with colour $i$, and next recolour every vertex in $N_{i} \cap \varphi^{-1}(\{i\})$ with colour $c$. Since the sets $\left(N_{i}\right)_{1 \leqslant i \leqslant \omega}$ are pairwise non-adjacent and we recolour an independent set in each set $N_{i}$, we obtain a proper colouring of $G$. And since $\chi(G)-1 \geqslant \omega$, because $\chi(G)>f(G) \geqslant \omega$, this colouring uses less than $\chi(G)$ colours, a contradiction. Therefore, the graph $G$ contains no triangle.

Consider a shortest cycle $C$ in $G$ (there is one since $\chi(G)>f(G) \geqslant 2$ ). By (B) and the previous argument, we have $|V(C)|=2 p+1$ where $p \geqslant 2$. Let $v_{1}, v_{2}, \ldots, v_{2 p+1}$ be the vertices on $C$, consecutively. Let $N_{i}^{\prime}$ be the set of neighbours of $v_{i}$ not in $C$. Note that every vertex not in $C$ has at most one neighbour in $C$, for otherwise $G$ would contain either an odd cycle shorter than $C$ or an induced even cycle, thereby contradicting (B). 
Consequently, the sets $\left(N_{i}^{\prime}\right)_{1 \leqslant i \leqslant 2 p+1}$ are pairwise disjoint. It also follows from (B) that the sets $\left(N_{i}^{\prime}\right)_{1 \leqslant i \leqslant 2 p+1}$ are pairwise non-adjacent.

Let $G_{C}=G-C$. By (A), we obtain a $(\chi(G)-2)$-colouring $\varphi$ of $G_{C}$. Similarly as before, we argue how to deduce from $\varphi$ a $(\chi(G)-1)$-colouring $\phi$ of $G$, hence obtaining the final contradiction. Recall that $\chi(G) \geqslant 4$. For $i \in\{1,2, \ldots, p\}$, we colour $v_{2 i}$ with colour 1 and $v_{2 i-1}$ with colour 2 . We colour $v_{2 p+1}$ with colour 3 . Set $c=\chi(G)-1$, so $c \notin \varphi\left(V\left(G_{C}\right)\right)$. For each $i \in\{1,2, \ldots, 2 p+1\}$, every vertex in $N_{i}^{\prime} \cap \varphi^{-1}\left(\phi\left(v_{i}\right)\right)$ is coloured with colour $c$. We define $\phi$ to be equal to $\varphi$ on all the remaining vertices of $G$. Similarly as before, the properties of the sets $\left(N_{i}^{\prime}\right)_{1 \leqslant i \leqslant 2 p+1}$ ensure that $\phi$ is a proper $(\chi(G)-1)$-colouring of $G$. This contradiction concludes the proof of the theorem.

\section{Conclusion}

With now a clear understanding of why Theorem 3 holds, it is tempting to look for a strengthening of the theorem. We already discussed in Section 1 some natural generalisations of the statement that unfortunately do not hold. In this section, we conclude this work with a discussion of other potential generalisations. Let us first restate Theorem 3 as follows, where the notation $H \subseteq_{i} G$ means that $H$ is an induced subgraph of $G$.

Theorem 3 (Folkman [Fol70]). For every graph $G$,

$$
\chi(G) \leqslant \max _{H \subseteq_{i} G}(|V(H)|-2 \cdot(\alpha(H)-1)) .
$$

The parameter $\alpha(H)$ can be interpreted as "the size of the largest induced subgraph of $H$ with chromatic number 1 ". This suggests a new approach for a generalisation. For every positive integer $p$, define $\alpha_{p}(H)$ as the size of a largest induced subgraph of $H$ with chromatic number at most $p$.

Question 5. Given a positive integer $p$, does there exist $c_{p}>1$ such that for every graph $G$,

$$
\chi(G) \leqslant \max _{H \subseteq_{i} G}\left(|V(H)|-c_{p} \cdot\left(\alpha_{p}(H)-p\right)\right) ?
$$

Note that the inequality holds for $c_{p} \leqslant 1$ by taking $H=G$. Theorem 3 answers the case $p=1$ of Question 5 in the affirmative, showing that $c_{1}$ can even be taken as high as 2. Before considering larger values of $p$, let us point out that $c_{1}=2$ is best possible. Indeed, suppose that $c_{1}>2$ and take $G$ to be the odd cycle $C_{5}$. Because $\chi\left(C_{5}\right)=3$ and $c_{1}>2$, the maximum in the right side of (2) must be attained by $H$ being the null graph (that is, $V(H)=\varnothing$ ), implying that $c_{1} \geqslant 3$. However, the Mycielski graph $M_{c_{1}^{\prime}}$, where $c_{1}^{\prime}=\left\lfloor c_{1}\right\rfloor$, now contradicts the desired inequality. Since $\chi\left(M_{c_{1}^{\prime}}\right)=c_{1}^{\prime}+1$, taking $H$ to be the null graph is not sufficient to ensure the inequality. Moreover, as $c_{1}^{\prime} \geqslant 3$ we know that $\operatorname{mir}\left(M_{c_{1}^{\prime}}\right) \geqslant \frac{1}{c_{1}^{\prime}}$ (as mentioned in the introduction), and hence $\alpha(H) \geqslant$ $|V(H)| / c_{1}^{\prime}$ whenever $H$ is a non-null induced subgraph of $M_{c_{1}^{\prime}}$. It follows that, in this case, $|V(H)|-c_{1}(\alpha(H)-1) \leqslant|V(H)|-c_{1}^{\prime}(\alpha(H)-1) \leqslant c_{1}^{\prime}<\chi\left(M_{c_{1}^{\prime}}\right)$, a contradiction. This shows that $c_{1}=2$ is best possible. 
For larger values of $p$, it turns out that the answer to Question 5 is always negative. First note that since $\alpha_{p+1}(H) \geqslant \alpha_{p}(H)+1$ in any graph $H$ with chromatic number higher than $p$, a negative answer for a positive integer $p_{0}$ implies a negative answer for every integer $p \geqslant p_{0}$. We now explain why the answer is negative for $p=2$.

For simplicity, we define $f_{2}(G)$ to be

$$
\max _{H \subseteq_{i} G}\left(|V(H)|-c_{2} \cdot\left(\alpha_{2}(H)-2\right)\right) .
$$

Let $\ell \geqslant 2$. For $k \geqslant 2$, let $M_{k, \ell}^{\prime}$ be obtained by applying $k-2$ times the Mycielski construction to the cycle on $2 \ell+1$ vertices. Note that $M_{k, 2}^{\prime}$ is the standard Mycielski graph $M_{k}$.

Suppose by contradiction that $c_{2}>1$. We prove by induction on $k$ that for every $k \geqslant 1$, we have $c_{2} \geqslant \frac{k+1}{2}$. For $k=1$, this is true by our assumption that $c_{2}>1$. For $k \geqslant 2$, we consider $f_{2}\left(M_{k, \ell}^{\prime}\right)$ for $\ell$ large enough in terms of $k$.

If the maximum in the definition of $f_{2}\left(M_{k, \ell}^{\prime}\right)$ is attained on the null graph, then we obtain $2 c_{2} \geqslant \chi\left(M_{k, \ell}^{\prime}\right)=k+1$ as desired. Thus we may assume this is not the case. If the maximum is attained on a proper non-null induced subgraph $H$, then $H$ is $k$-colourable and thus satisfies $\alpha_{2}(H) \geqslant \frac{2|V(H)|}{k}$. Since $H$ gives a higher value than that given by the null graph in the definition of $f_{2}\left(M_{k, \ell}^{\prime}\right)$, we deduce that

$$
|V(H)|-c_{2} \cdot\left(\alpha_{2}(H)-2\right)>2 c_{2} .
$$

However, using that $c_{2} \geqslant \frac{k}{2}$ (by induction), we obtain $\alpha_{2}(H)<\frac{2|V(H)|}{k}$, a contradiction.

It follows that the maximum in the definition of $f_{2}\left(M_{k, \ell}^{\prime}\right)$ is attained by $M_{k, \ell}^{\prime}$ itself. Observe that $\left|M_{k, \ell}^{\prime}\right|=(\ell+1) \cdot 2^{k-1}-1$ and $\alpha_{2}\left(M_{k, \ell}^{\prime}\right)=\ell \cdot 2^{k-1}$. Consequently,

$$
f_{2}\left(M_{k, \ell}^{\prime}\right)=(\ell+1) \cdot 2^{k-1}-1-c_{2} \cdot\left(\ell \cdot 2^{k-1}-2\right) \geqslant \chi\left(M_{k, \ell}^{\prime}\right)=k+1,
$$

which does not hold for $\ell$ large enough in terms of $k$, since $c_{2}>1$. Therefore, this last case cannot occur either, and $c_{2} \geqslant \frac{k+1}{2}$ holds, as announced.

In summary, we have shown that $c_{2}>1$ implies that $c_{2} \geqslant \frac{k+1}{2}$ for every positive integer $k$, and hence that $c_{2}$ is unbounded, a contradiction. We conclude that $c_{2} \leqslant 1$ must hold.

Given this state of affairs, it was suggested to one of the authors to try and replace $\alpha_{2}(H)$ with the maximum size of an induced subgraph of $H$ isomorphic to a bipartite cograph. However, the answer remains negative even when considering the maximum size of an induced subgraph of $H$ isomorphic either to a complete bipartite graph with one side of size at most 2 or to $P_{1}+P_{2}$, the graph on three vertices with only one edge. To see this, it suffices to apply Mycielski's construction once on a large clique, and to do the arithmetic for various cases depending on the structure of a subgraph attaining maximum potential (whether it contains more vertices of the clique than of the maximum stable set, and whether it contains the unique vertex whose neighbourhood is a stable set). While this supersedes the above argument that $c_{2} \leqslant 1$, the proof is decidedly non-illuminating, and we refrain from including it here. Considering how restricted the replacement for $\alpha_{2}(H)$ is here, generalising Theorem 3 in this direction does not seem feasible either. 
Our original motivations for finding a short proof of Theorem 3 were to make sense of the statement and understand what bigger truth it could be part of. With the new-found insight, Theorem 3 seems all the more to be a truly isolated statement, almost a singularity in the realm of graph colouring.

\section{Acknowledgements}

This research was started at the Graph Theory meeting in Oberwolfach in January 2019. Thanks to the organizers and to the other workshop participants for creating a productive working atmosphere. Thanks to Paul Seymour for stimulating discussions on this topic.

\section{References}

[AKS80] Miklós Ajtai, János Komlós, and Endre Szemerédi. A note on Ramsey numbers. J. Combin. Theory Ser. A, 29(3):354-360, 1980.

[AKS81] Miklós Ajtai, János Komlós, and Endre Szemerédi. A dense infinite Sidon sequence. European J. Combin., 2(1):1-11, 1981.

[CGL06] Mathew Cropper, András Gyárfás, and Jenő Lehel. Hall ratio of the Mycielski graphs. Discrete Math., 306(16):1988-1990, 2006.

[EH68] Pál Erdős and András Hajnal. Problem 3. Theory of Graphs, Proc. Colloq. Tihany, Hungary 1966, page 362, 1968.

[Fol70] Jon H. Folkman. An upper bound on the chromatic number of a graph. In Combinatorial theory and its application, II, (Proc. Colloq., Balatonfüred, 1969), pages 437-457. North-Holland, Amsterdam, 1970.

[Gri83] Jerrold R. Griggs. An upper bound on the Ramsey numbers $R(3, k)$. J. Combin. Theory Ser. A, 35(2):145-153, 1983.

[Gyá97] András Gyárfás. Fruit salad. Electron. J. Combin., 4(1):Research Paper 8, 1997.

[Haj65] András Hajnal. A theorem on k-saturated graphs. Canad. J. Math., 17:720-724, 1965.

[Kim95] Jeong Han Kim. The Ramsey number $R(3, t)$ has order of magnitude $t^{2} / \log t$. Random Structures Algorithms, 7(3):173-207, 1995.

[LPU95] Michael Larsen, James Propp, and Daniel Ullman. The fractional chromatic number of Mycielski's graphs. J. Graph Theory, 19(3):411-416, 1995.

[Ree99] Bruce Reed. Mangoes and blueberries. Combinatorica, 19(2):267-296, 1999.

[REG08] Research Experiences for Graduate Students (REGS), 2008. University of Illinois. See https://faculty.math.illinois.edu/ west/regs/folkman.html.

[She91] James B. Shearer. A note on the independence number of triangle-free graphs. II. J. Combin. Theory Ser. B, 53(2):300-307, 1991. 